\title{
Cost effectiveness of a government supported policy strategy to decrease sodium intake: global analysis across 183 nations
}

\author{
Michael Webb, ${ }^{1}$ Saman Fahimi, ${ }^{2}$ Gitanjali M Singh, ${ }^{3}$ Shahab Khatibzadeh, ${ }^{2}$ Renata Micha, ${ }^{3}$ \\ John Powles, ${ }^{4}$ Dariush Mozaffarian ${ }^{3}$
}

'Stanford University, Stanford, CA, USA, and Institute for Fiscal Studies, London, UK

${ }^{2}$ Harvard TH Chan School of

Public Health, Boston, MA, USA

${ }^{3}$ Tufts Friedman School of

Nutrition Science \& Policy, 150

Harrison Ave, Boston, MA

02111, USA

${ }^{4}$ Cambridge Institute of Public Health, Cambridge, UK

Correspondence to:

D Mozaffarian

dariush.mozaffarian@tufts.edu

Additional material is published online only. To view please visit

the journal online.

Cite this as: BMJ 2017;356:16699

http://dx.doi.org/10.1136/bmj.i6699

Accepted: 01 December 2016

\section{ABSTRACT}

\section{OBJECTIVE}

To quantify the cost effectiveness of a government policy combining targeted industry agreements and public education to reduce sodium intake in 183 countries worldwide.

DESIGN

Global modeling study.

SETTING

183 countries.

POPULATION

Full adult population in each country.

INTERVENTION

A "soft regulation" national policy that combines targeted industry agreements, government monitoring, and public education to reduce population sodium intake, modeled on the recent successful UK program. To account for heterogeneity in efficacy across countries, a range of scenarios were evaluated, including $10 \%, 30 \%$, $0.5 \mathrm{~g} /$ day, and $1.5 \mathrm{~g} /$ day sodium reductions achieved over 10 years. We characterized global sodium intakes, blood pressure levels, effects of sodium on blood pressure and of blood pressure on cardiovascular disease, and cardiovascular disease rates in 2010, each by age and sex, in 183 countries. Country specific costs of a sodium reduction policy were estimated using the World Health Organization Noncommunicable Disease Costing Tool. Country specific impacts on mortality and disability adjusted life years (DALYs) were modeled using comparative risk assessment. We only evaluated program costs, without incorporating potential healthcare savings from prevented events, to provide conservative estimates of cost effectiveness

MAIN OUTCOME MEASURE

Cost effectiveness ratio, evaluated as purchasing power parity adjusted international dollars (equivalent to the country specific purchasing power of US\$) per DALY saved over 10 years.

\section{WHAT IS ALREADY KNOWN ON THIS TOPIC}

In prior research in a limited number of high income nations, national policies to reduce excess sodium intake have been estimated to be highly cost effective for reducing hypertension and cardiovascular disease

For most countries, the cost effectiveness of a national policy intervention to reduce sodium intake is unknown

\section{WHAT THIS STUDY ADDS}

We found that a government "soft regulation" strategy combining targeted industry agreements and public education to reduce population sodium intake by $10 \%$ over 10 years would be extremely cost effective in nearly all of 183 nations evaluated

This would result in an average cost effectiveness ratio (not accounting for potential healthcare savings from averted events) of $1 \$ 204 /$ disability adjusted life year

\section{RESULTS}

Worldwide, a $10 \%$ reduction in sodium consumption over 10 years within each country was projected to avert approximately 5.8 million DALYs/year related to cardiovascular diseases, at a population weighted mean cost of $I \$ 1.13$ per capita over the 10 year intervention. The population weighted mean cost effectiveness ratio was approximately I\$204/DALY. Across nine world regions, estimated cost effectiveness of sodium reduction was best in South Asia ( $\$ 116$ / DALY); across the world's 30 most populous countries, best in Uzbekistan (I\$26.08/DALY) and Myanmar (I\$33.30/DALY). Cost effectiveness was lowest in Australia/New Zealand (I\$880/DALY, or $0.02 \times$ gross domestic product (GDP) per capita), although still substantially better than standard thresholds for cost effective (<3.0×GDP per capita) or highly cost effective (<1.0×GDP per capita) interventions. Most (96.0\%) of the world's adult population lived in countries in which this intervention had a cost effectiveness ratio $<0.1 \times$ GDP per capita, and $99.6 \%$ in countries with a cost effectiveness ratio $<1.0 \times$ GDP per capita.

\section{CONCLUSION}

A government "soft regulation" strategy combining targeted industry agreements and public education to reduce dietary sodium is projected to be highly cost effective worldwide, even without accounting for potential healthcare savings.

\section{Introduction}

Excessive sodium consumption is common and linked to cardiovascular burdens in most countries. Overall, 181 of 187 countries, representing $99.2 \%$ of the global adult population, have mean sodium intakes exceeding the World Health Organization recommended maximum of $2 \mathrm{~g} /$ day. ${ }^{1}$ Based on this threshold, an estimated 1648000 annual deaths from cardiovascular diseases worldwide were attributable to excess dietary sodium in $2010 .^{2}$ Accordingly, the 2013 United Nations' Global Action Plan for the Prevention and Control of Noncommunicable Diseases has prioritized sodium reduction as one of nine key targets for all member nations in 2013-20. ${ }^{3}$

A potential barrier for implementation of this recommendation is cost. Many countries have limited resources for health interventions, requiring careful assessment of costs and cost effectiveness. Several countries now have national programs that include a specific aim of reducing population sodium intake; for instance, as of 2012, 29 European nations, consisting of all EU Member States as well as Norway and Switzerland, had salt reduction initiatives in place. ${ }^{4}$ Yet the cost effectiveness of such efforts globally is uncertain. While prior studies have estimated sodium reduction 
policies to be highly cost effective, or even cost saving, in specific countries, the potential cost effectiveness of such strategies has been analyzed for only a handful of nations and regions, mostly focused on high income nations, and in ways that are not generally comparable. .-14 $^{-14}$

To address this key gap in knowledge, we assessed the cost effectiveness of sodium reduction strategies in 183 nations, based on the most up to date available data on age specific and sex specific sodium intakes, blood pressure levels, and cardiovascular disease burdens worldwide, the dose-response effects of sodium on blood pressure and of blood pressure on cardiovascular disease, and nation specific costs for each component of the intervention. Together, these allowed us to model and estimate, using comparable and consistent methods, the cost effectiveness of sodium reduction strategies for every country.

\section{Methods}

\section{Sodium reduction intervention}

We modeled the effects and costs of a 10 year government "soft regulation" policy to reduce population sodium consumption (see supplementary eTable 1 for details of the model assumptions). The intervention program was modeled on recent experience in the $\mathrm{UK}^{15}$ and included government supported industry agreements to reduce sodium in processed foods, government monitoring of industry compliance, and a public health campaign targeting consumer choices. In the UK, for example, this intervention was based on collaboration between national government offices focused on nutrition (Food Standards Agency) and health (ministers of public health), together with non-governmental advocacy organizations (Consensus Action on Salt \& Health). The program applied sustained pressure on food manufacturers to pursue progressive reformulation, reinforced by food group specific targets, independent monitoring, and a sustained media campaign against excess salt intake. The program we modeled was thus more robust and costly than simple "voluntary reformulation."

We assumed the intervention would scale up linearly over 10 years, with one 10th of the total sodium reduction in the first year, two 10ths in the second, and so on, reaching full efficacy in the final year. We recognized that alternative programs, such as mandatory regulation, would likely have larger effects, reduce sodium consumption more quickly, and at lower cost, but may be less politically feasible in many countries.

\section{Intervention costs}

Country specific resource needs and costs were derived using the WHO-CHOICE database, ${ }^{16}$ which includes detailed component specific estimates of inputs (ingredients) required for each intervention stage for each country's government and the estimated unit price for each input in that country including for example costs of human resources, training, meetings, supplies, equipment, and mass media (see supplementary eMethods). To facilitate comparisons between countries, we converted all costs to international dollars (I\$) (see supplementary eMethods), which account for each nation's currency as well as purchasing power parity. ${ }^{17}$
One I\$ in any given country can be interpreted as the funds needed to purchase the same amounts of goods or services in that country as one US\$ would purchase in the US. For countries with lower incomes than the US, conversion of our findings from I\$ to US\$ would substantially increase the apparent cost effectiveness (ie, the cost in US\$ per disability adjusted life year (DALY) saved would be much lower). We summed costs by year to calculate the total cost of the 10 year intervention for each country, with $3 \%$ annual discounting.

We modeled only governmental intervention costs, for several reasons. First, this cost is most relevant to budget constrained governments, since the program cost must be borne directly and immediately. Second, net industry sector costs for product reformulation in each country would be difficult to determine because once the relevant reformulation has been undertaken in any single country, the knowledge of that reformulation can be extended with much less additional cost to other countries. For example, multinational companies transfer improved recipes and reformulation strategies across borders with no cost, as do food scientists moving between firms, and so on. Third, in contrast to recent US models, ${ }^{1011}$ we did not include estimated healthcare savings or increased productivity from prevented cardiovascular disease events because such savings could, in theory, be partly offset by new downstream health events resulting from enhanced survival ${ }^{18} 19$ and because comparable healthcare and productivity costing data are available for a minority of countries globally. Because including such cost savings would be optimal according to many cost effectiveness guidelines, our results for overall cost effectiveness should be considered a conservative estimate.

\section{Heterogeneity in intervention costs and effectiveness}

Though the WHO costing framework already accounted for some sources of variation by country in terms of resources required and nation specific costs, we recognized that details of planning, development, and implementation might further vary from country to country beyond what is captured by the costing tool. We also recognized that achieved effectiveness would vary from country to country. Our base model assumed an average cost of this framework (already adjusted for in-country differences in resource use and costs, according to the WHO costing tool), and an average effectiveness. To understand the robustness of our findings to these assumptions, we tested widely varying costs-including variations in resource use and cost of between 0.25 -fold and fivefold the base-and varying intervention effectiveness, including $10 \%$ and $30 \%$ proportional reductions and $0.5 \mathrm{~g} /$ day and $1.5 \mathrm{~g} /$ day absolute reductions in sodium intake over 10 years. Plausible intervention effectiveness was informed by experiences in the UK, which achieved a $14.7 \%$ ( $0.6 \mathrm{~g} /$ day $)$ reduction in population sodium intake over 10 years, ${ }^{20}$ and Turkey, which reported a more rapid 16\% (1.2 g/day) reduction over four years. ${ }^{21}$ Together, these findings provided a broad range of possible scenarios against which to evaluate the cost effectiveness of the intervention. 


\section{Intervention impact on DALYs}

Using data on population demographics, sodium consumption, blood pressure levels, and rates of cardiovascular disease, each in 26 strata by age and sex within each country, ${ }^{2}$ we estimated the number of disability adjusted life years that would be averted by the intervention in each country for each year between 2011 and 2020. Risk reduction in each age-sex-country stratum was calculated from the effect of sodium reduction on systolic blood pressure, including variation in this effect by age, race, and hypertensive status; and the effect of blood pressure reduction on cardiovascular disease, including variation in this effect by age. ${ }^{2}$ The final comparative risk assessment model incorporated each of these sources of heterogeneity, as well as their uncertainty. Stratum specific effects, accounting for underlying demographics and baseline cardiovascular disease rates, were summed to derive national (or regional) effects (see supplementary eMethods for details on these inputs and their modeling).

While some prior observational studies suggest a J-shaped relation between sodium intake and cardiovascular disease, ${ }^{22}$ this could be explained by potential biases of sodium assessment in observational studies (see supplementary eMethods). ${ }^{23}$ In extended follow-up of sodium reduction trials that overcame many of these limitations, linear risk reductions were seen, including lower risk with intakes less than $2.3 \mathrm{~g} /$ day. $^{24}$ We recognized that while the precise optimal level of sodium intake remains controversial, every major national and international organization that has reviewed all the evidence has concluded that high sodium intake increases cardiovascular disease risk and that lowering sodium intake reduces such risk, with optimal identified intakes ranging from less than $1.2 \mathrm{~g} /$ day to less than $2.4 \mathrm{~g} /$ day. $^{2}$ We used an optimal intake of $2.0 \mathrm{~g} /$ day (WHO) for our main analysis. For any sodium reductions below this level, we modeled neither additional benefit nor risk, consistent with recent Institute of Medicine conclusions. ${ }^{25}$ In sensitivity analyses, we also evaluated lower (1.0 g/day) and higher (3.0 g/ day) thresholds for optimal intake.

Our modeling further utilized known strengths of blood pressure as "an exemplar surrogate endpoint for cardiovascular mortality and morbidity."26 Prospective cohort studies suggest log-linear associations between systolic blood pressure and cardiovascular disease events, down to around $110 \mathrm{~mm} \mathrm{Hg}^{27}$; and randomized controlled trials indicate that benefits of blood pressure lowering interventions are largely proportional to the magnitude of blood pressure reduction, rather than the specific intervention, with similar proportional reductions in cardiovascular disease events down to pretreatment blood pressures of around $110 \mathrm{~mm} \mathrm{Hg} \cdot{ }^{27-29} \mathrm{In}$ our model, we assumed a log-linear dose-response between blood pressure and cardiovascular disease until a systolic blood pressure level of $115 \mathrm{~mm} \mathrm{Hg}$, below which we assumed no further lowering of risk. Given the relatively rapid reductions in cardiovascular disease events in randomized trials of blood pressure lowering therapies, and the prolonged period of our intervention (10 years), we did not model any lag and assumed concurrent gradual benefits in both blood pressure reduction and cardiovascular disease.

\section{Cost effectiveness ratios}

To calculate the cost effectiveness ratio for each country, we divided the total effect on DALYs by the total cost of the intervention over 10 years. We compared these cost effectiveness ratios to WHO benchmarks, which define a cost effectiveness ratio <3×gross domestic product (GDP) per capita as cost effective, and $<1 \times$ GDP per capita as highly cost effective. ${ }^{30}$ We appreciated the potential limitations of these WHO benchmarks ${ }^{31}$ yet also their practicality for multinational studies such as this. To quantify statistical uncertainty, we used probabilistic sensitivity analyses based on 1000 Monte Carlo simulations to derive $95 \%$ uncertainty intervals, with varying inputs for sodium use, blood pressure levels, effects of sodium on blood pressure, and effects of blood pressure on cardiovascular disease (see supplementary eMethods).

\section{Patient involvement}

No patients were involved in setting the research question or the outcome measures, nor were they involved in developing plans for design or implementation of the study. No patients were asked to advise on interpretation or writing up of results. There are no plans to disseminate the results of the research to study participants or the relevant patient community.

\section{Results}

Cost effectiveness of sodium reduction by national income level and region

Taking into account local prices, currencies, and purchasing power, the relative contributions of each intervention component to the total 10 year cost differed appreciably between countries (see supplementary eFigure 1). For instance, costs of supplies and equipment, meetings, and training were uniformly low (averaging I\$0.01 per capita, I\$0.01 per capita, and I\$0.04 per capita, respectively), whereas costs of human resources and mass media were much higher and more variable across countries. Globally, average purchasing power parity adjusted costs for human resources (personnel salaries) were I\$0.27 per capita, but with a ninefold range comparing high income (I\$0.93) with low income (I\$0.10) countries. Human resources were most costly in Australia/New Zealand (I\$1.26 per capita), Western Europe (I\$1.03), and Canada/US (I\$0.82); and lowest in South Asia (I\$0.06). Mass media costs were generally the most expensive component of the intervention: I\$0.80 per capita globally, I\$1.07 for high income nations, and I\$0.44 for low income nations. They represented the most costly component of the intervention in every region except for Australia/New Zealand, Canada/US, and Western Europe, where human resources was the most costly component.

Globally, the estimated average cost effectiveness ratio of the 10 year intervention was approximately I\$204 per DALY saved (95\% uncertainty interval I $\$ 149$ to I\$322) (table 1). This did not include potential savings 


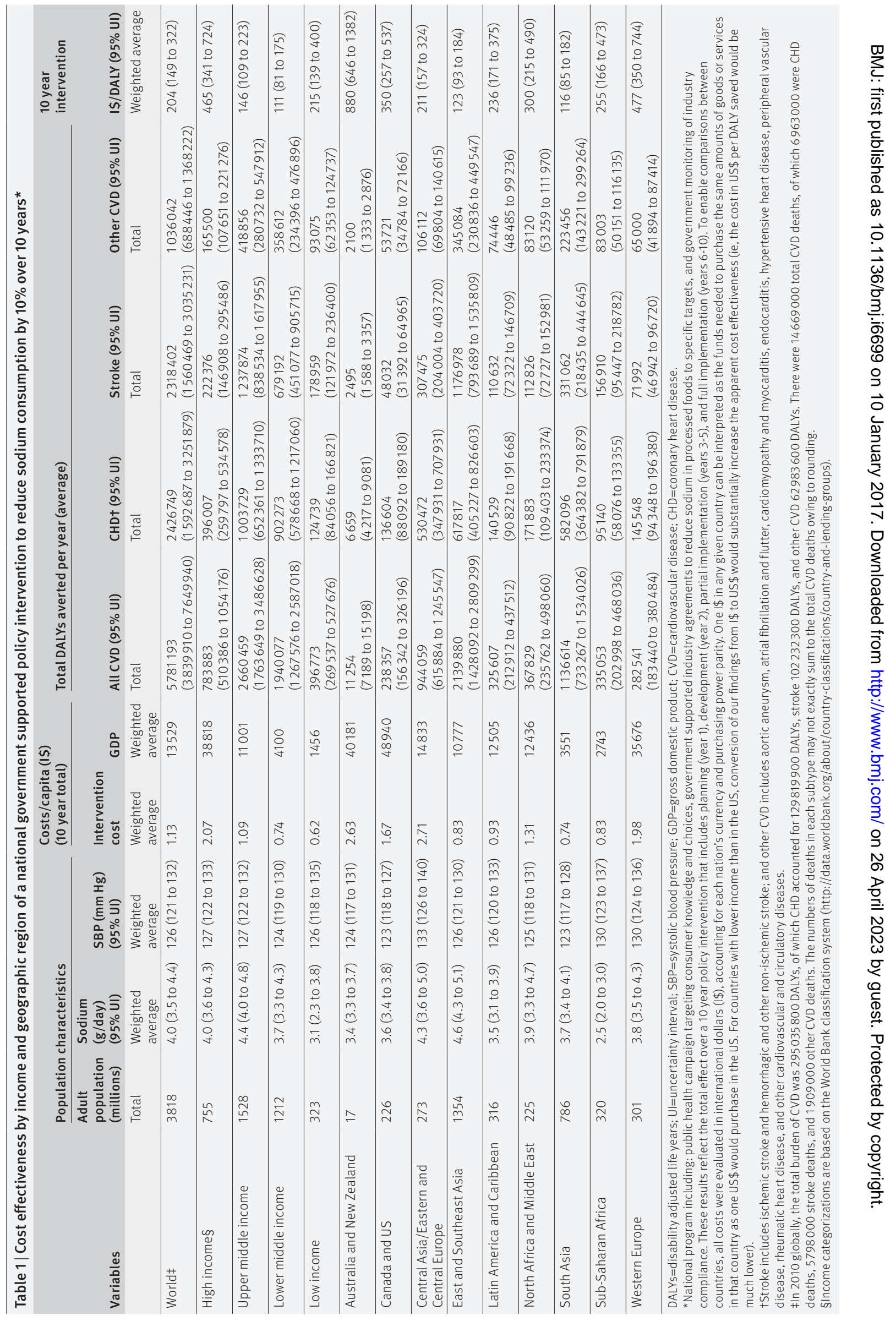


from lower healthcare costs or higher productivity owing to averted cardiovascular disease events, which would each further improve the estimated cost effectiveness. The estimated cost effectiveness ratio was lowest (best) in lower middle income (I\$111, I\$81 to I\$175) and upper middle income countries (I\$146, I\$109 to I\$223), higher in low income countries (I\$215, I\$139 to I\$400), and highest in high income countries (I\$465, I $\$ 341$ to I\$724). By region, the lowest cost effectiveness ratios were in South Asia and East/Southeast Asia (I\$116 and I\$123, respectively). In Central Asia/Eastern and Central Europe, high intervention efficacy partly offset its higher projected cost, generating the next best cost effectiveness ratio (I\$211, I\$157 to I\$324).

\section{Effectiveness, cost, and cost effectiveness by country}

Across individual countries, the estimated intervention efficacy, in terms of DALYs averted per 1000 people, was highest in Kazakhstan (23.0, 95\% uncertainty interval 15.6 to 29.8), Georgia (21.6, 14.3 to 28.3), Belarus (19.8,

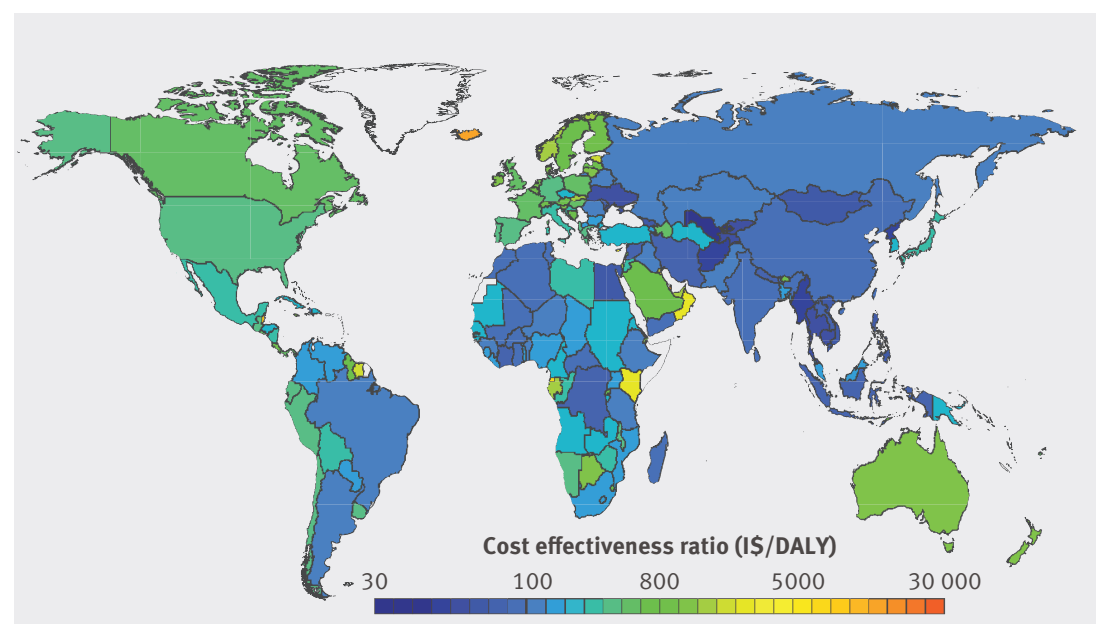

Fig 1 | Cost effectiveness (purchasing power adjusted I\$ / disability adjusted life year) by country of a national policy intervention to reduce sodium consumption by $10 \%$

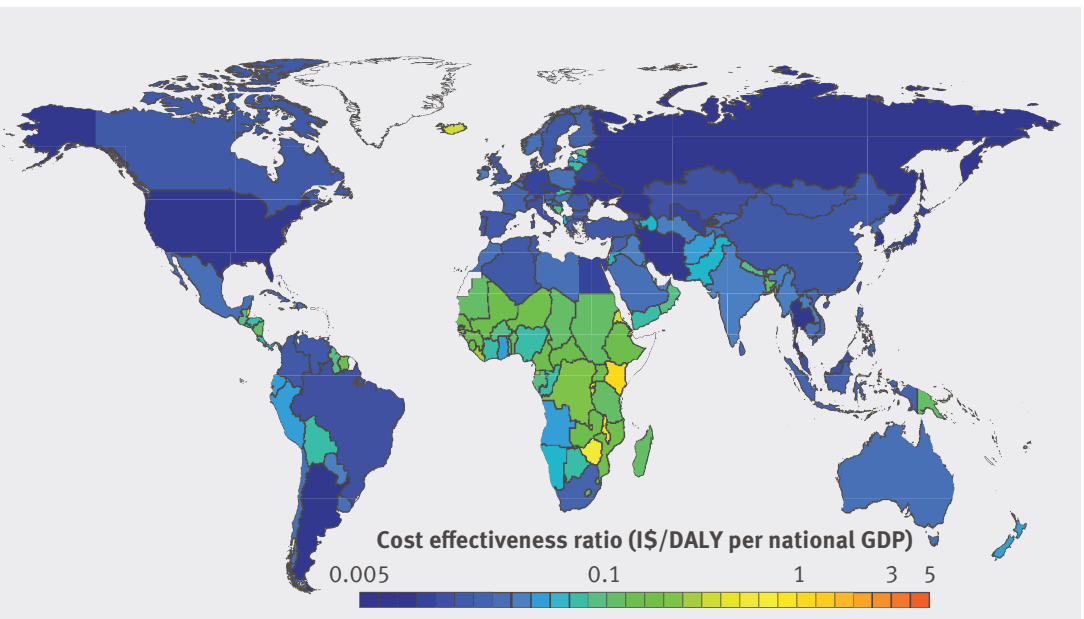

Fig 2 | Cost effectiveness (purchasing power adjusted I\$/disability adjusted life year (DALY) as a multiple of gross domestic product (GDP) per capita) by country of a national policy intervention to reduce sodium consumption by $10 \%$
12.8 to 26.9$)$, Ukraine (19.0, 12.3 to 25.9$)$, Mongolia (18.9, 12.1 to 25.0 ), and Russia (18.8, 12.2 to 25.5 ) (see supplementary eTable 3 ). The relative rankings of these nations should be considered in the context of the uncertainty in the estimates that preclude, for example, confirming statistically significant differences in efficacy between Kazakhstan and Russia. Yet, the range of estimated efficacy across the 183 nations was large-for example, compared with the countries above, much lower in Jamaica (1.9, 1.1 to 2.7), Qatar (1.4, 0.8 to 1.9), Rwanda (1.3, 0.6 to 2.3), and Kenya (0.4, 0.2 to 0.7).

Per capita, estimated 10 year intervention cost was lowest in Myanmar, Vietnam, Democratic People's Republic of Korea (each I\$0.31), Thailand (I\$0.33), Nepal (I\$0.40), and Uzbekistan (I\$0.41) (see supplementary eTable 3). A total of 68 countries had estimated 10 year intervention costs of less than I $\$ 1.00$ per capita. For 84 countries, estimated costs were between I $\$ 1.00$ and I\$9.99, for 19 countries, between I\$10 and I\$29.99, and for 12, greater than I $\$ 30$.

Estimated national cost effectiveness ratios were correspondingly variable (fig 1). Uzbekistan's was lowest (best) at I\$26.08/DALY (95\% uncertainty interval 20.08 to 39.02), followed by Myanmar (I\$33.30, 25.10 to 50.46). Twenty eight countries had estimated cost effectiveness ratios below I $\$ 100 /$ DALY, and 112 more, below I $\$ 1000 /$ DALY. Eleven nations, all small, had estimated cost effectiveness ratios between I\$10000 and I\$30 000/ DALY (see supplementary eTable 3).

\section{WHO benchmarks for cost effectiveness}

In comparison with WHO benchmarks (cost effectiveness ratio $<3 \times$ GDP per capita is cost effective, $<1 \times$ GDP per capita, highly cost effective), ${ }^{30}$ the 10 year sodium reduction intervention was estimated to be highly cost effective globally. Across all 183 countries, the estimated cost effectiveness ratio of this policy intervention was $>3 \times$ GDP per capita in only one nation (Marshall Islands: $4.7 \times$ GDP per capita), between $3 \times$ GDP per capita and $1 \times$ GDP per capita in six nations (Kenya, Tonga, Kiribati, Samoa, Micronesia, Comoros), and highly cost effective in all other nations (fig 2). Indeed, in 130 countries, representing more than $96 \%$ of the world's population, the estimated cost effectiveness ratio was $<0.1 \times$ GDP per capita, far below usual cost effectiveness thresholds. This included each of the world's 20 most populous countries (fig 3).

\section{Potential heterogeneity of effectiveness and costs} A national policy intervention to reduce sodium intake remained highly cost effective globally and by world region when we considered alternative effectiveness (proportional reduction of $30 \%$, absolute reduction of $0.5 \mathrm{~g} /$ day or $1.5 \mathrm{~g} /$ day); and alternative thresholds of optimal intake (the level at which further sodium reduction produces no further health benefits) of $3.0 \mathrm{~g} /$ day or $1.0 \mathrm{~g} /$ day (table 2). Generally, achieving larger sodium reduction targets (eg, 30\%, $1.5 \mathrm{~g} /$ day) was more cost effective (see supplementary eFigure 2), but even modest achieved reductions ( $10 \%$ or $0.5 \mathrm{~g} /$ day over 10 years) were highly cost effective. Under any of these scenarios, 


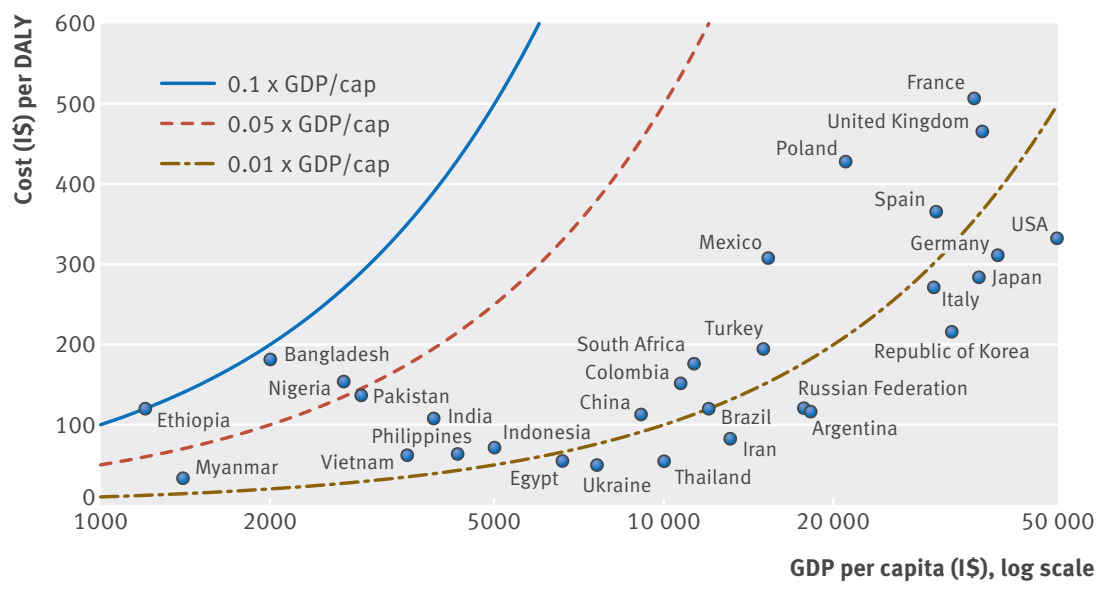

Fig 3 Affordability of a national policy intervention to reduce sodium consumption by $10 \%$ in the world's 20 most populous countries. Each point represents the cost effectiveness of the intervention (I\$/disability adjusted life year (DALY)) for a given country against that country's gross domestic product (GDP) per capita (I\$), adjusted for purchasing power. The lines represent $0.01 \times, 0.05 \times$, and $0.1 \times$ GDP per capita, selected as reasonable fractions against which to compare our estimates of affordability. Notably, each of these thresholds is substantially lower than the World Health Organization benchmarks for an intervention being cost effective (<3.0 $\times$ GDP per capita) or highly cost effective (<1.0×GDP per capita). For example, Nigeria and Bangladesh, being to the right of the blue line and to the left of the red dotted line, have a cost effectiveness ratio less than $0.1 \times$ GDP per capita but greater than $0.05 \times$ GDP per capita intake, even if the intervention costs were fivefold greater than the baseline estimate, $96 \%$ of the world's population would live in countries with a cost effectiveness ratio $<0.5 \times$ GDP per capita; and for a $30 \%$ reduction in sodium intake, $99 \%$ would.

\section{Discussion}

We found that a government "soft regulation" policy intervention to reduce national sodium consumption by $10 \%$ over 10 years was projected to be highly cost effective in nearly every country in the world ( $<1 \times$ gross domestic product (GDP) per capita per disability life year (DALY) saved), and remarkably cost effective $(<0.05 \times$ GDP per capita per DALY) in most countries. Hundreds of thousands of deaths, and millions of DALYs, were estimated to be potentially averted annually, at low cost.

\section{Comparison with other prevention strategies}

These cost effectiveness ratios compare very favorably with other prevention strategies. For example, "best buy" pharmacologic interventions to reduce cardiovascular disease in high income countries have much higher estimated cost effectiveness ratios, such as \$21000/DALY or more for primary prevention with statin drugs and \$6000/DALY or more for secondary prevention with $\beta$ blockers. ${ }^{32} 33$ By contrast, for this national government supported intervention to reduce sodium intake by $10 \%$ over 10 years, we project an average cost effectiveness ratio of I\$465/DALY in high income countries. Similarly, our projected cost effectiveness ratio of I\$143/DALY in low income and middle income countries compares favorably with an estimated cost effectiveness ratio of I\$900/DALY for a cardiovascular disease combination pill ("polypill”) targeted at high risk people in developing countries. ${ }^{34}$ Notably, most of these prior pharmacologic cost effectiveness ratios included estimated health savings from averted cardiovascular disease events, which produces substantially more favorable cost effectiveness ratios than if estimated health savings are omitted, as in our analysis. ${ }^{33} 34$

Our novel results, together with prior studies in selected countries, ${ }^{5-14}$ provide evidence that a national policy for reduction in sodium intake is highly cost effective, and substantially more so than even highly cost effective medical prevention strategies. This advantage likely arises from several factors. This policy is relatively inexpensive to implement, utilizing system wide "soft regulation" rather than provision of individual level medical care. It also decreases cardiovascular risk at a population level, such that even small changes in distributions of risk factors translate into large clinical benefits, ${ }^{35}$ as compared with more intensive strategies delivered only to a subset of people. Thus, there are meaningful "returns to scale" on both the cost side and the effect side. This suggests that a national reduction in sodium intake is a "best buy" for governments, deserving careful consideration for adoption by countries worldwide.

Despite differences in modeling methods, other studies of sodium reduction interventions in selected 


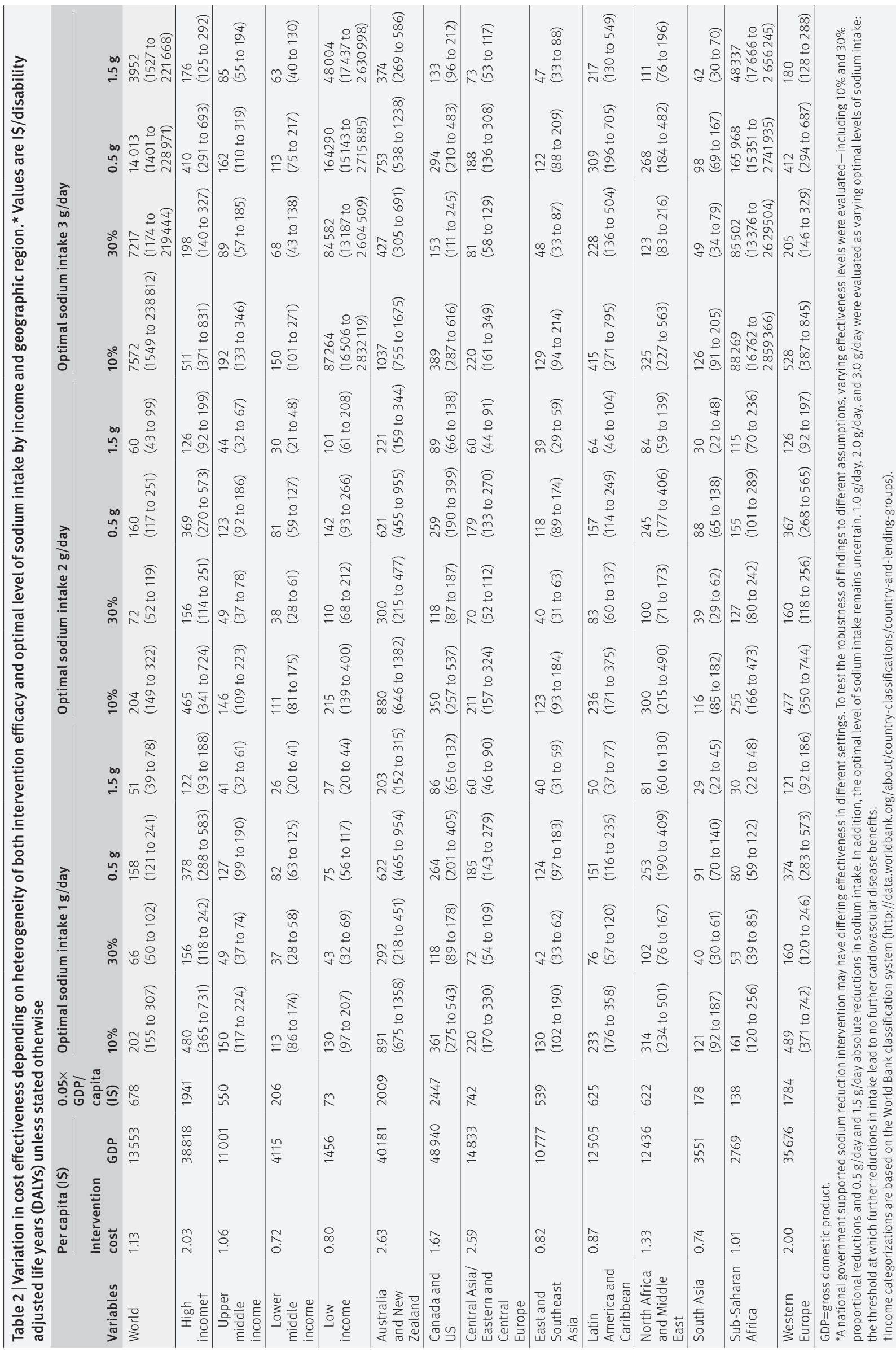


nations have also found them to be extremely cost effective. ${ }^{59-1113}$ Many of these prior analyses incorporated estimated health system savings from averted cardiovascular disease events, which generally rendered the interventions not only cost effective but also actually cost saving-that is, with dominant cost effectiveness ratios less than zero. For example, one analysis in the US estimated that a $0.4 \mathrm{~g} /$ day (about $11 \%$ ) sodium reduction over 10 years would save from $\$ 4 \mathrm{bn}$ to $\$ 7 \mathrm{bn}$ in healthcare costs. ${ }^{10}$ Some analyses further accounted for productivity gains from reduced morbidity and mortality from cardiovascular disease, further increasing cost savings. Investigations that, like ours, calculated only intervention costs and DALYs averted, without including any estimates of health system savings, arrived at similar cost effectiveness ratios for comparable regions (eg, I\$561 for western Europe ${ }^{36}$ versus our cost effectiveness ratio of I\$477 in that region).

Our investigation builds on and substantially extends such prior analyses of potential sodium reduction interventions in several important respects. First, most included only a single high income nation. ${ }^{5101113}$ One prior analysis included 23 more varied nations but only estimated averted deaths, rather than DALYs, ${ }^{7}$ preventing comparison with other cost effectiveness ratios. In contrast with prior analyses, we also jointly incorporated heterogeneity in blood pressure effects of sodium reduction by age, race, and hypertensive status, providing more accurate estimates for the impact on cardiovascular disease. Additionally, our analysis of 183 countries using consistent methods enabled us to explore sources of heterogeneity and sensitivity in estimated cost effectiveness across diverse nations and regions.

\section{Sources of heterogeneity}

Differences in intervention costs were one of the major drivers of varying cost effectiveness ratios. The large variation of human resource and mass media costs across countries suggests potential savings from multinational efforts to reduce sodium intake, which could benefit from economies of scale. For instance, the new European Union Salt Reduction Framework, which monitors national sodium reduction initiatives and supports implementation efforts across multiple member nations, ${ }^{4}$ could be emulated elsewhere. Consistent with the relevance of scale, the 20 countries with highest per capita intervention costs all had national populations of less than 500000 adults. The higher cost of mass media, compared with other intervention components, further suggests a need for research on how best to target such resources. The recent finding ${ }^{37}$ that salt reduction in the UK arose largely from product reformulation rather than changes in consumer choice suggests that, in countries where most dietary sodium comes from processed food (eg, $77 \%$ in the US ${ }^{38}$ ), the robustness and compliance with industry targets may be more relevant than mass media components. On the other hand, public awareness of sodium in foods and health effects could be essential for generating sufficient public and policy maker pressure on industry to meet stated targets. In nations with lower proportions of manufactured food, industry focused efforts might lead to smaller absolute reductions in sodium intake. Yet many such countries also have lower baseline levels of sodium consumption, ${ }^{1}$ so that proportional reductions might be similar. In comparison, for certain Asian nations such as China, substantial amounts of sodium are added at home, making education and media efforts more relevant. Nevertheless, even with an up to fivefold increase in total costs, our multinational investigation suggests that a government supported program to reduce sodium intake would be highly cost effective for nearly every country in the world.

Our findings were robust to differing thresholds for optimal sodium intake. While the precise optimal level of sodium intake remains uncertain, ${ }^{25}$ to our knowledge ours is the first cost effectiveness analysis to evaluate the relevance of this uncertainty to policy. We found that this threshold influences relative cost effectiveness only in countries with the lowest intakes, with little effect in most others. For example, cost effectiveness ratios increase notably in Sub-Saharan Africa when the threshold is raised from $2.0 \mathrm{~g} / \mathrm{day}$ to $3.0 \mathrm{~g} / \mathrm{day}$, but relatively little in most other nations (table 2).

\section{Strengths and limitations of this study}

Our analysis has several strengths. The model used comparable and consistent methods to estimate cost effectiveness in 183 countries, including contemporary data on age, sex, and nation specific distributions of sodium consumption, blood pressure, and rates of cardiovascular disease. Blood pressure effects of sodium reduction were derived from meta-analysis of randomized trials, accounting for differences by age, race, and hypertension; and the cardiovascular effects of blood pressure lowering from pooled analysis of prospective studies, accounting for age. The modeled intervention included a realistic scale-up trajectory and target sodium reduction. The cost estimates incorporated country specific demographic, economic, and health data, together with results from cross country nontraded input price regressions, to produce credible estimates of national prices. We incorporated uncertainty in multiple input parameters (measures of sodium exposure, distributions of blood pressure, effects of sodium on blood pressure, effects of blood pressure on cardiovascular disease) by multi-way probabilistic Monte Carlo simulations, and additional uncertainty in intervention effectiveness and intervention costs by separate sensitivity analyses.

Potential limitations should be considered. The estimates of sodium consumption, blood pressure levels, and rates of cardiovascular disease were based on raw data covering most but not all of the global population, with hierarchical estimation of the remainder. ${ }^{13940}$ Our estimates of health benefits accounted only for cardiovascular disease, whereas high sodium intake is also associated with vascular stiffness, renal dysfunction, and stomach cancer, independent of blood pressure levels. ${ }^{41-43}$ We did not account for possible unintended consequences of the intervention, such as changes in 
population choices of overall foods consumed. We did not model health system savings from averted cardiovascular disease events. Better cardiovascular health may produce compression of disease and costs into the last years of life, reducing overall morbidity and lifetime costs, but modeling such potential health transitions and treatment costs for every nation globally is not yet feasible. We did not evaluate potential effects on disparities within countries; for instance, food product reformulation to reduce sodium intake in England has been estimated to reduce socioeconomic inequalities in cardiovascular disease. ${ }^{44}$ Our models are based on a 10 year intervention period including planning, development, and staged implementation. Over the longer term, intervention costs may decrease, while lifetime health benefits might also increase. Thus, these findings should be considered a platform on which to base intermediate term policies, recognizing that longer term effectiveness should also be evaluated. Our assumptions about intervention implementation may differ in various real world situations, producing larger or smaller costs and effect sizes. However, our analyses of the sensitivity of our findings to variations in costs and effectiveness demonstrated that overall cost effectiveness was highly robust to alternative assumptions. We did not evaluate other potential strategies to reduce sodium intake, such as mandatory quality standards, taxation, complementary state or community initiatives, or multi-component approaches, such as seen in Japan and Finland. ${ }^{45-47}$ These might produce similar or even greater reductions in sodium intake at less cost, but are also perhaps less feasible in certain nations.

\section{Conclusions}

Even without incorporating potential healthcare savings from averted events, we found that a government supported, coordinated national policy to reduce population sodium intake by $10 \%$ over 10 years would be cost effective in all and extremely cost effective in nearly all of 183 nations evaluated.

\section{We thank the World Health Organization for use of the} Noncommunicable Disease Costing Tool, and the Institute for Health Metrics and Evaluation for use of their cardiovascular disease DALY estimates.

Contributors: MW and DM conceptualised the study and wrote the final draft of the paper; DM also provided funding support and supervision. MW undertook the analysis and wrote the first draft of the paper. SF, GMS, SK, and RM prepared data and commented on the paper. JP commented on the paper. All authors approved the final version. MW acts as guarantor of the study.

Funding: This research was supported by the National Heart, Lung, and Blood Institute (R01 HL115189; principal investigator DM) and the National Institute of Diabetes and Digestive and Kidney Diseases (T32 training grant in academic nutrition, DK007703; GMS), National Institutes of Health. The sponsors had no role in the study design; in the collection, analysis, and interpretation of data; in the writing of the report; and in the decision to submit the article for publication.

Competing interests: All authors have completed the ICMJE uniform disclosure form at www.icmje.org/coi_disclosure.pdf and declare: financial support from the National Institutes of Health for the submitted work. DM reports ad hoc honorariums or consulting fees from Boston Heart Diagnostics, Haas Avocado Board, Astra Zeneca, GOED, DSM, and Life Sciences Research Organization, none of which were related to topics of dietary sodium. The other authors report no financial relationships with any organizations that might have an interest in the submitted work in the previous three years.
Ethical approval: Not required

Data sharing: The global data on sodium intake may be requested from the authors for academic collaborations; see www. globaldietarydatabase.org/requesting-data.html. Global data on blood pressure is available for download at www1.imperial.ac.uk/ publichealth/departments/ebs/projects/eresh/majidezzati/ healthmetrics/metabolicriskfactors/metabolic_risk_factor_maps/ Global data on cardiovascular events is available for download from the Global Burden of Diseases Study at http://ghdx.healthdata.org/ global-burden-disease-study-2013-gbd-2013-data-downloads.

Transparency: The lead author (MW) affirms that the manuscript is an honest, accurate, and transparent account of the study being reported; that no important aspects of the study have been omitted; and that any discrepancies from the study as planned (and, if relevant, registered) have been explained.

This is an Open Access article distributed in accordance with the Creative Commons Attribution Non Commercial (CC BY-NC 3.0) license, which permits others to distribute, remix, adapt, build upon this work non-commercially, and license their derivative works on different terms, provided the original work is properly cited and the use is noncommercial. See: http://creativecommons.org/licenses/by-nc/3.0/.

1 Powles J, Fahimi S, Micha R, et al. Global Burden of Diseases Nutrition and Chronic Diseases Expert Group (NutriCoDE). Global, regional and national sodium intakes in 1990 and 2010: a systematic analysis of $24 \mathrm{~h}$ urinary sodium excretion and dietary surveys worldwide.BMJ Open 2013;3:e003733. doi:10.1136/ bmjopen-2013-003733.

2 Mozaffarian D, Fahimi S, Singh GM, et al. Global Burden of Diseases Nutrition and Chronic Diseases Expert Group. Global sodium consumption and death from cardiovascular causes.N EnglJ Med 2014;371:624-34. doi:10.1056/NEJMoa1304127.

3 Lichtenstein AH, Ausman LM, Carrasco W, Jenner JL, Ordovas JM, Schaefer EJ. Hypercholesterolemic effect of dietary cholesterol in diets enriched in polyunsaturated and saturated fat. Dietary cholesterol, fat saturation, and plasma lipids. Arterioscler Thromb 1994;14:168-75. doi:10.1161/01.ATV14.1.168.

4 European Commission. Survey on Member States' Implementation of the EU Salt Reduction Framework. http://ec.europa.eu/health/ nutrition_physical_activity/docs/salt_report1_en.pdf (accessed 28 May, 2016).

5 Selmer RM, Kristiansen IS, Haglerod A, et al. Cost and health consequences of reducing the population intake of salt. J Epidemiol Community Health 2000:54:697-702. doi:10.1136/jech.54.9.697.

6 Abelson P. Returns on investment in public health: an epidemiological and economic analysis prepared for the Department of Health and Ageing.Department of Health and Ageing, 2001.

7 Asaria P, Chisholm D, Mathers C, Ezzati M, Beaglehole R. Chronic disease prevention: health effects and financial costs of strategies to reduce salt intake and control tobacco use.Lancet 2007;370:2044-53. doi:10.1016/S0140-6736(07)61698-5.

8 Penz ED, Joffres MR, Campbell NR. Reducing dietary sodium and decreases in cardiovascular disease in Canada. Can / Cardiol 2008:24:497-1. doi:10.1016/S0828-282X(08)70625-1.

9 Palar K, Sturm R. Potential societal savings from reduced sodium consumption in the U.S. adult population.Am J Health Promot 2009;24:49-57. doi:10.4278/ajhp.080826-QUAN-164.

10 Bibbins-Domingo K, Chertow GM, Coxson PG, et al. Projected effect of dietary salt reductions on future cardiovascular disease. N Engl / Med 2010;362:590-9. doi:10.1056/NEJMoa0907355.

11 Smith-Spangler CM, Juusola JL, Enns EA, Owens DK, Garber AM. Population strategies to decrease sodium intake and the burden of cardiovascular disease: a cost-effectiveness analysis. Ann Intern Med 2010;152(8):481-7, W170-3

12 Cobiac LJ, Vos T, Veerman JL. Cost-effectiveness of interventions to reduce dietary salt intake.Heart 2010;96:1920-5. doi:10.1136/ hrt.2010.199240.

13 Barton P. Andronis L, Briggs A, McPherson K, Capewell S. Effectiveness and cost effectiveness of cardiovascular disease prevention in whole populations: modelling study.BM/ 2011;343:d4044. doi:10.1136/bmj. d4044.

14 Bertram MY, Steyn K, Wentzel-Viljoen E, Tollman S, Hofman KJ. Reducing the sodium content of high-salt foods: effect on cardiovascular disease in South Africa. S Afr Med J 2012;102:743-5. doi:10.7196/SAMJ.5832.

15 UK Food Standards Agency. UK Salt Reduction Initiatives. www.food. gov.uk/sites/default/files/multimedia/pdfs/saltreductioninitiatives. pdf (accessed 28 May, 2016).

16 Johns B, Baltussen R, Hutubessy R. Programme costs in the economic evaluation of health interventions. Cost EffResour Alloc 2003;1:1 doi:10.1186/1478-7547-1-1.

17 Summers R, Heston A. The Penn World Table (Mark 5): an expanded set of international comparisons, 1950-1987. Q J Econ 1991;106:327-68doi:10.2307/2937941. 
18 Meltzer D. 42 Future costs in medical cost-effectiveness analysis. The Elgar companion to health economics 2012:447

19 Weinstein MC, Siegel JE, Gold MR, Kamlet MS, Russell LB. Recommendations of the Panel on Cost-effectiveness in Health and Medicine. JAMA 1996;276:1253-8. doi:10.1001/jama.1996.03540150055031.

20 Sadler K, Nicholson S, Steer T, et al. National Diet and Nutrition Survey - Assessment of dietary sodium in adults (aged 19 to 64 years) in England, 2011.UK Department of Health, 2012.

21 News WHO. Progress in reducing salt consumption in Turkey. www euro.who.int/en/what-we-do/health-topics/disease-prevention/ nutrition/news/news/2013/04/progress-in-reducing-saltconsumption-in-turkey (accessed 28 May, 2016).

22 O’Donnell M, Mente A, Rangarajan S, et al. PURE Investigators. Urinary sodium and potassium excretion, mortality, and cardiovascular events[Online First: Epub Date] |.].N Engl J Med 2014;371:612-23. doi:10.1056/NEJMoa1311889.

23 Cobb LK, Anderson CA, Elliott P, et al. American Heart Association Council on Lifestyle and Metabolic Health. Methodological issues in cohort studies that relate sodium intake to cardiovascular disease outcomes: a science advisory from the American Heart Association.Circulation 2014;129:1173-86. doi:10.1161/CIR.0000000000000015.

24 Cook NR, Appel LJ, Whelton PK. Lower levels of sodium intake and reduced cardiovascular risk. Circulation 2014;129:981-9. doi:10.1161/ CIRCULATIONAHA.113.006032.

25 Institute of Medicine. Sodium Intake in Populations: Assessment of Evidence.The National Academies Press, 2013.

26 Institute of Medicine. Evaluation of Biomarkers and Surrogate Endpoints in Chronic Disease. National Academies Press, 2010.

27 Lewington S, Clarke R, Qizilbash N, Peto R, Collins R. Prospective Studies Collaboration. Age-specific relevance of usual blood pressure to vascular mortality: a meta-analysis of individual data for one million adults in 61 prospective studies. Lancet 2002;360:1903-13. doi:10.1016/S0140-6736(02)11911-8.

28 Williamson JD, Supiano MA, Applegate WB, et al. SPRINT Research Group. Intensive vs Standard Blood Pressure Control and Cardiovascular Disease Outcomes in Adults Aged $\geq 75$ Years: A Randomized Clinical Trial. JAMA 2016;315:2673-82. doi:10.1001/jama.2016.7050.

29 Xie X, Atkins E, Lv J, et al. Effects of intensive blood pressure lowering on cardiovascular and renal outcomes: updated systematic review and meta-analysis.Lancet 2016;387:435-43. doi:10.1016 S0140-6736(15)00805-3.

30 WHO Commission on Macroeconomics and Health. Macroeconomics and health: Investing in health for economic development. World Health Organization, 2001

31 Marseille E, Larson B, Kazi DS, Kahn JG, Rosen S. Thresholds for the cost-effectiveness of interventions: alternative approaches.Bull World Health Organ 2015;93:118-24. doi:10.2471/BLT.14.138206.

32 Goldman L, Sia ST, Cook EF, Rutherford JD, Weinstein MC. Costs and effectiveness of routine therapy with long-term beta-adrenergic antagonists after acute myocardial infarction. N Engl / Med 1988;319:152-7. doi:10.1056/NEJM198807213190306.

33 Goldman L, Weinstein MC, Goldman PA, Williams LW. Costeffectiveness of HMG-CoA reductase inhibition for primary and secondary prevention of coronary heart disease. JAMA 1991:265:1145-51. doi:10.1001/jama.1991.03460090093039.
34 Gaziano T. Prevention and treatment of chronic diseases in developing countries. Expert Paper No. 2011/2: UN Dept of Economic and Social Affairs Population Division, 2011.

35 Rose G. Sick individuals and sick populations. Int J Epidemio 2001;30:427-32, discussion 433-4. doi:10.1093/ije/30.3.427.

36 Murray CI, Lauer JA, Hutubessy RC, et al. Effectiveness and costs of interventions to lower systolic blood pressure and cholesterol: a global and regional analysis on reduction of cardiovascular-disease risk.Lancet 2003;361:717-25. doi:10.1016/S0140-6736(03)12655-4.

37 Griffith R, O'Connell M, Smith K. The importance of product reformulation versus consumer choice in improving diet quality. IFS Working Paper W14/15, 2014

38 Mattes RD, Donnelly D. Relative contributions of dietary sodium sources. J Am Coll Nutr 1991;10:383-93. doi:10.1080/07315724.1991.10718167

39 Danaei G, Finucane MM, Lin JK, et al. Global Burden of Metabolic Risk Factors of Chronic Diseases Collaborating Group (Blood Pressure). National, regional, and global trends in systolic blood pressure since 1980: systematic analysis of health examination surveys and epidemiological studies with 786 country-years and 5.4 million participants.Lancet 2011;377:568-77. doi:10.1016/ S0140-6736(10)62036-3

40 Murray CJ, Vos T, Lozano R, et al. Disability-adjusted life years (DALYS) for 291 diseases and injuries in 21 regions, 1990-2010: a systematic analysis for the Global Burden of Disease Study 2010. Lancet 2012;380:2197-223. doi:10.1016/S0140-6736(12)61689-4.

41 Aburto NJ, Ziolkovska A, Hooper L, Elliott P, Cappuccio FP, Meerpohl IJ. Effect of lower sodium intake on health: systematic review and meta-analyses.BMJ 2013;346:f1326. doi:10.1136/bmj.f1326.

42 Graudal NA, Hubeck-Graudal T, Jurgens G. Effects of low sodium diet versus high sodium diet on blood pressure, renin, aldosterone, catecholamines, cholesterol, and triglyceride.Cochrane Database Syst Rev 2011;(11):CD004022. doi:10.1002/14651858.CD004022.pub3.

43 Joossens JV, Hill MJ, Elliott P, et al. European Cancer Prevention (ECP) and the INTERSALT Cooperative Research Group. Dietary salt, nitrate and stomach cancer mortality in 24 countries. Int J Epidemiol 1996;25:494-504. doi:10.1093/ije/25.3.494.

44 Gillespie DO, Allen K, Guzman-Castillo M, et al. The Health Equity and Effectiveness of Policy Options to Reduce Dietary Salt Intake in England: Policy Forecast.PLoS One 2015;10:e0127927. doi:10.1371/ journal pone.0127927.

45 Christoforou A, Trieu K, Land MA, Bolam B, Webster J. State-level and community-level salt reduction initiatives: a systematic review of global programmes and their impact.J Epidemiol Community Health 2016;70:1140-50. doi:10.1136/jech-2015-206997.

46 Trieu K, Neal B, Hawkes C, et al. Salt Reduction Initiatives around the World - A Systematic Review of Progress towards the Global Target. PLoS One 2015;10:e0130247. doi:10.1371/journal.pone. 0130247.

47 Hyseni L, Elliot-Green A, Lloyd-Williams F, et al. Systematic review of dietary salt reduction policies: evidence for an effectiveness hierarchy?[abstract]. Circulation 2016;133(Suppl 1):154

Supplementary information: Supplementary material 